\title{
VALIDITY OF QUANTUM ELECTRODYNAMICS AT SMALL DISTANCES
}

\author{
J. G. Asbury,* W. K. Bertram, $†$ U. Becker, P. Joos, M. Rohde, and A. J. S. Smith* \\ Deutsches Elektronen-Synchrotron, Hamburg, Germany
}

and

\author{
S. Friedlander, C. Jordan, and C. C. Ting $\dagger$ \\ Department of Physics, Columbia University, New York, New York \\ (Received 7 November 1966)
}

We have measured the yield of wide-angle electron-positron pairs produced in the reaction

$$
\gamma+\mathbf{C} \rightarrow e^{+}+e^{-}+\mathbf{C}
$$

in order to test the validity of quantum electrodynamics (QED) at small distances. The experiment, performed at the Deutsches Elektronen-Synchrotron (DESY) 6.2-GeV electron synchrotron, used a symmetrical magnetic spectrometer and counter techniques to detect the pairs. The results show that first-order QED correctly predicts the $e^{+} e^{-}$pair yield for momentum transfers to the virtual electron up to $400 \mathrm{MeV} / c$, in contradiction to earlier results where large deviations have been reported. ${ }^{1}$

The major contribution to Reaction (1), the Bethe-Heitler terms, have been calculated by Bjorken, Drell, and Frautschi (BDF). ${ }^{2}$ The Compton term is estimated to contribute less than a few percent to the yield. ${ }^{3}$ The symmetrical nature of the detector eliminates the contribution from interference between the BetheHeitler and Compton diagrams. At symmetry, if $E_{+}$is the energy of the positron and $\theta$ is its production angle, the momentum transfer to the virtual electron is given by $t^{2}=2 E_{+}{ }^{2} \theta^{2}$, while the momentum transfer $q$ to the recoil nucleus is given by $q^{2}=E_{+}{ }^{2} \theta^{4}$. Under the kinematical conditions of this experiment, $t \leqslant 400 \mathrm{MeV} / c$ and $\left\langle q^{2}\right\rangle^{1 / 2} \leqslant 50 \mathrm{MeV} / c$. Because $q$ is small, a heavy nuclear target may be used; the yield is proportional to $Z^{2}$, and very little correction is necessary for the nuclear form factors. ${ }^{4}$ The accuracy with which the experimental yield may be compared with QED is limited to a few percent by one's inability to calculate the Compton term exactly, and by the lack of precise measurements of the inelastic form factors of the target nucleus.

Experimental arrangement. - The apparatus is shown in Fig. 1. The bremsstrahlung beam is produced when the DESY circulating electron beam strikes an internal rotating target. The average photon intensity is $3 \times 10^{10}$ equivalent quanta per second; the duty cycle $2-4 \%$. The beam is defined by two lead collimators $10 \times 10$ $\mathrm{mm}^{2}$ and $15 \times 15 \mathrm{~mm}^{2}$, and cleared of charged particles by three dipoles. The first two dipoles bend horizontally, the third vertically. Approximately $35 \mathrm{~m}$ downstream from the rotating target a $2.5-\mathrm{g} / \mathrm{cm}^{2}$-thick carbon target was mounted on a calibrated optical bench. ${ }^{\mathbf{5}}$ At the target position the beam spot is approximately square in shape, $2.5 \mathrm{~cm} \times 2.5 \mathrm{~cm}$. The beam intensity is measured by a Wilson-type quantameter, which was calibrated in an external electron beam against a Faraday cup. ${ }^{6}$ The photons travel to the quantameter inside a vacuum pipe encased throughout its length by heavy concrete and lead.

The spectrometer itself consists of dipole magnets $\left(\mathrm{M}_{D}, \mathrm{M}_{A}, \mathrm{M}_{B}\right)$, scintillation counters $\left(\mathrm{L}_{1}, \mathrm{~L}_{2}, \mathrm{~L}_{3}, \mathrm{~L}_{4}, \mathrm{R}_{1}, \mathrm{R}_{2}, \mathrm{R}_{3}, \mathrm{R}_{4}\right)$, shower counters (SLC, SRC), threshold Cherenkov counters (LC, RC, HL, HR), and scintillationcounter hodoscopes (TL, TR, QL, QR, VL, VR). The magnet $M_{D}$ separates charged particles from the $\gamma$ beam and also sweeps very low-energy particles out of the system. Particles with a central spectrometer momentum $p_{0}$ are bent an angle of $15^{\circ}-\theta$ by $\mathrm{M}_{D}$, where $\theta$ is the horizontally projected production angle with respect to the $\gamma$ beam. After passing through $\mathrm{M}_{D}$, the central-momentum particles are bent a constant $-8^{\circ}$ by the $M_{B}$ located 2.18 $\mathrm{m}$ downstream from the center of $\mathrm{M}_{D}$. The target position and the field of $\mathrm{M}_{D}$ are chosen such that the trajectory of the central ray (cen-

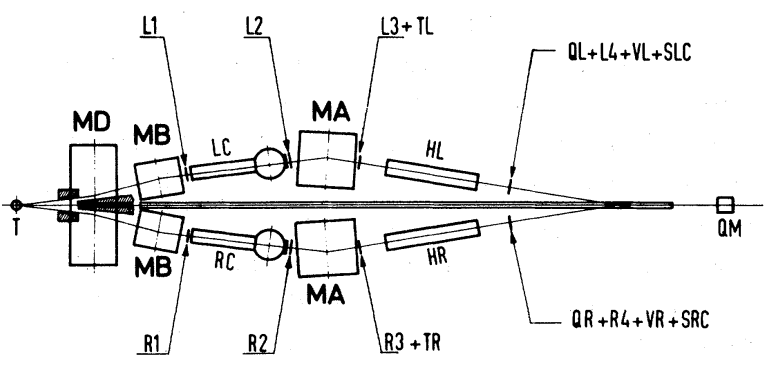

FIG. 1. Plan view of the spectrometer. 
tral momentum $p_{0}$ and angle $\theta_{0}$ ) after it enters the $\mathrm{M}_{B}$ 's is identical for all settings of the spectrometer. The magnets $\mathrm{M}_{A}, 5.39 \mathrm{~m}$ downstream from the $\mathrm{M}_{B}$ 's, then bend the central ray a constant angle $-17.47^{\circ}$. This arrangement has the following properties essential to the experiment:

(1) The acceptance of the spectrometer is not limited by the edges of magnets or by shielding, being defined instead by the scintillation trigger counters $\mathrm{L}_{2}-\mathrm{L}_{4}, \mathrm{R}_{2}-\mathrm{R}_{4}$. All counters are located such that their surfaces are not directly exposed to the target. The instantaneous rate in $\mathrm{L}_{2}$ and $\mathrm{R}_{2}$, the "hottest" of the triggering counters, is always $<3 \mathrm{Mc} / \mathrm{sec}$.

(2) The spread in position and angle of the particles as they pass through all the threshold and shower counters is nearly independent of the spectrometer setting. Therefore, any slight inefficiency of these counters cannot lead to a momentum-transfer-dependent effect.

(3) The spectrometer recombines rays of constant $p \theta \sim t$ and, therefore, has a large acceptance and at the same time a good $t$ resolution. For a given spectrometer setting, the acceptance is $\delta p / p \simeq \pm 0.18, \delta \theta / \theta \simeq \pm 0.14, \delta t / t$ $\simeq \pm 0.10$, and $\delta \psi= \pm 8 \mathrm{mrad}$, where $\psi$ is the projected vertical production angle. ${ }^{7}$

The efficiency of all counters was measured in a $1-\mathrm{GeV}$ electron beam. Each trigger and hodoscope counter was $>99.9 \%$ efficient over its entire area. For the angular acceptance needed in this experiment, the efficiency of all four threshold counters and both shower counters was $>99 \%$. During the experiment the pion rejection ${ }^{8}$ of the threshold and shower counters was constantly monitored. Even under the highest instantaneous rates encountered, the combined rejection of the pair ( $\mathrm{LC}$, RC) was always $>4 \times 10^{3}$, of (HL, HR) always $>1 \times 10^{3}$, and of (SLC, SRC) always $>100$.

Logic circuits capable of operating at 125 $\mathrm{Mc} / \mathrm{sec}$ were used to minimize dead time and accidentals as well as the following corrections to the master rate $M=\left(\mathrm{R}_{2}+\mathrm{R}_{3}+\mathrm{R}_{4}+\mathrm{RC}+\mathrm{SRC}\right.$, $\left.\mathrm{L}_{2}+\mathrm{L}_{3}+\mathrm{L}_{4}+\mathrm{LC}+\mathrm{SLC}\right)$ :

(1) Random accidentals. Because the singlearm electron rate was as much as 2000 times higher than the electron-pair rate, the contribution of random coincidences had to be monitored and kept lower than a few percent. For this reason, the incident beam intensity was always kept low enough so that the rates of coincidence circuits with resolutions of 16,12 , $8,7,6,5$, and $4 \mathrm{nsec}$ between the two arms never exceed each other by more than $5 \%$. Normally, for $(2-3) \times 10^{10}$ equivalent quanta/ sec incident photon intensity the rates differed by $<1 \%$. Therefore, the random accidentals in the rate $M$ are $<1 \%$ and can be calculated from the relative rates of these circuits.

(2) Pion contamination. The ratio of pion pairs to electron pairs was in some cases as high as 1000 to 1 . Although the requirements for $M$ provide a pion rejection $>10^{5}$, we used the counters HL and HR to monitor the adequacy of this rejection. Coincidences MHL (MHR) were made between $M$ and $H L$ (HR), and finally the coincidence MC was made between MHL and MHR. The rates of M, MHL, MHR, and MC all agreed to within $1 \%$ throughout the experiment. We are thus certain that the data are not contaminated with pions; if they were, the additional pion rejection of HL and HR would cause the rates MHL, MHR, and MC to be significantly lower than $M$.

(3) Dead time. We monitored each circuit with a fast scaler, and kept the beam intensity low enough such that the total dead time of the system was normally $2-3 \%$, and never more than $5 \%$.

The stability of all counting rates was constantly checked during the experiment, and all counter voltages were kept constant to within $\pm 10 \mathrm{~V}$. The left-arm rate agreed with the right-arm rate to within a few percent, and the reproducibility of the $e^{+} e^{-}$rate was better than $3 \%$ over a two-month running period.

Analysis. - The theoretical $e^{+} e^{-}$yields were calculated by two independent methods. The first was a conventional Monte Carlo technique whereby events with randomly generated production angles, momenta, and target positions were exposed to the magnetic fields and apertures of the system. In this manner the average cross section, the spectrometer acceptance, and various kinematical quantities were simultaneously calculated. Corrections for bremsstrahlung and multiple scattering in the target and along the spectrometer were included in the calculation. The decrease in the theoretical yield due to bremsstrahlung, while significant, varied little over the spectrometer settings, ranging from 32 to $37 \%$. The effect of multiple scattering, a decrease in the theoretical yield, was always less than $12 \%$. Essential to the Monte Carlo integration was the accurate determination of the magnet transport equations. Because of the large momentum 
range accepted, neither first- nor second-order transport theory could be used. Instead, the equations were determined by numerically integrating a family of 40 trajectories through a grid of the measured field values of each magnet (these fields were known to 3 parts in $10^{4}$ ). The transport coefficients were then obtained from the trajectories by a least-squares method. The transport equations included all terms linear, bilinear, and pure quadratic in $x, x^{\prime}$, $z, z^{\prime}$, and $\delta p / p$ (except those excluded by symmetry) and terms up to fourth order in $(\delta p / p) /$ $(1+\delta p / p)$. Comparing these coefficients with those obtained from first- and second-order theory, we found good agreement, any differences being attributable to the greater accuracy of the fourth-order calculation. A sufficient number of events were treated by the Monte Carlo technique to determine the theoretical yield within an uncertainty of $\pm 2.5 \%$.

In the second method by which the yield was calculated, each magnet was assumed to have a uniform field over its effective length; ray tracing was then used to determine the acceptance-limiting trajectories. An accurate approximation to $d \sigma_{\mathrm{BH}}{ }^{9}$ was then numerically integrated over the acceptance window so obtained. The acceptance and average cross sections agreed in all cases with those of the Monte Carlo calculation to within $5 \%$. As a further check, we averaged the exact cross section as given by Drell and Walecka ${ }^{10}$ over the spectrometer acceptances, finding agreement with BDF to better than $1 \%$.

Many experimental checks were made to ensure that the spectrometer behaved as designed:

(1) We found that the experimental yield at low momentum transfer agrees with the prediction of QED.

(2) Wire-orbit measurements performed on both spectrometer arms agreed with the calculated central angles and momenta to within $0.5 \%$, the accuracy limit of the wire-orbit technique.

(3) To test our treatment of multiple scattering and bremsstrahlung, we placed in each arm of the spectrometer (in front of $L_{2}$ and $R_{2}$ ) a $0.63-\mathrm{cm}$-thick Lucite sheet large enough to cover the spectrometer aperture. For $p_{0}=2250$, $\theta_{0}=4^{\circ}$, a $20 \pm 5 \%$ decrease in the yield was observed, in good agreement with the calculated decrease.

(4) At a production angle of $4^{\circ}$ and a maximum bremsstrahlung energy $k_{\max }=3.05 \mathrm{GeV}$, the $e^{+} e^{-}$yield was measured as a function of the spectrometer momentum setting over the momentum range 1.167-2.000 GeV/c. At the upper end of this momentum range, where the bremsstrahlung spectrum cuts off, the yield depends very sensitively upon the spectrometer acceptance. The measured and calculated yields were in good agreement, showing that the acceptance had been accurately calculated.

(5) To check the properties of the spectrometer and of the programs used, the distributions of electron-pair events were measured by the hodoscopes. In a typical data run, with central angle $4^{\circ}$, central momentum $1.830 \mathrm{GeV} / c$, the distribution shown in Fig. 2 was obtained in the hodoscope QL, the expected and observed distributions being in good agreement. Similar results were obtained for the other hodoscopes.

(6) The electron-pair rate did not change when the shielding inside the gap of $\mathbf{M}_{D}$ was moved either toward or away from the accepted region. This is good evidence that the shielding is not a source of background events. Checks (3)-(5) above indicate that scattering from $\mathrm{M}_{B}$ and $\mathrm{M}_{A}$ pole faces also did not contribute to the measured yield.

Results and conclusion. - Half the data were taken at each polarity of the spectrometer to eliminate interference between the Compton and Bethe-Heitler processes. Corrections were made for the dead time of the electronics, accidentals, beam attenuation in the target, target-out rates, multiple Coulomb scattering,

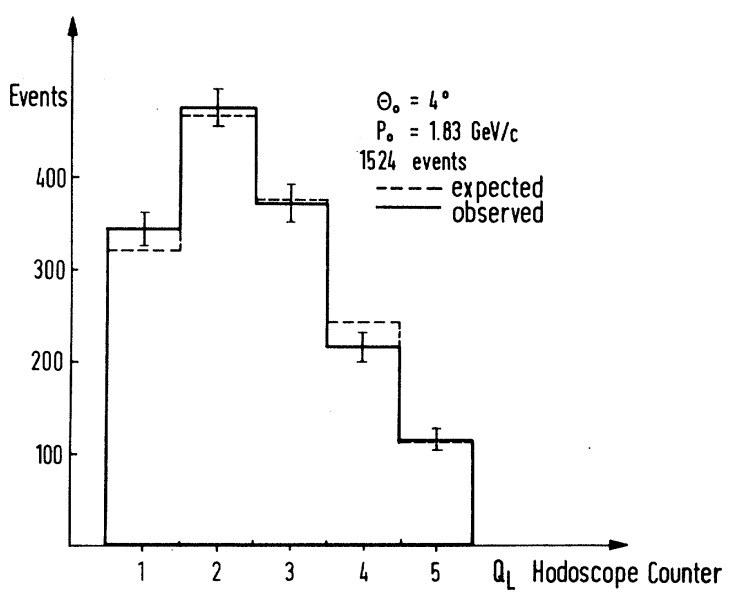

FIG. 2. Comparison of calculated and observed event distributions in the $Q_{L}$ hodoscope counters, for central spectrometer angle $\theta_{0}=4^{\circ}$ and momentum $p_{0}=1.83$ $\mathrm{GeV} / c$. 
and bremsstrahlung losses along the spectrometer. Approximately $25 \%$ of the running time was spent measuring target-out rates. These rates were found to be nearly independent of angle and momentum, never exceeding $10 \%$ of the rates with the target in place. As discussed earlier, pion contamination was measured to be always less than $1 \%$. In calculating the theoretical yield, we used analytical expressions for the nuclear form factors, ${ }^{11}$ but did not consider contributions from inelastic carbon form factors or from the Compton term. The total radiative correction (including corrections for hard photon emission) is almost constant over the various spectrometer settings, decreasing the theoretical yield by $(3.0 \pm 1.0) \%$.

In order to eliminate possible systematic errors, the data points were chosen in the 4 $\times 4$ matrix form shown in Fig. 3(a). The errors shown are statistical, including those introduced by the subtraction of the target-out rates. At least 400 events were accumulated at each point, most points having $\approx 1000$ events. In each col-

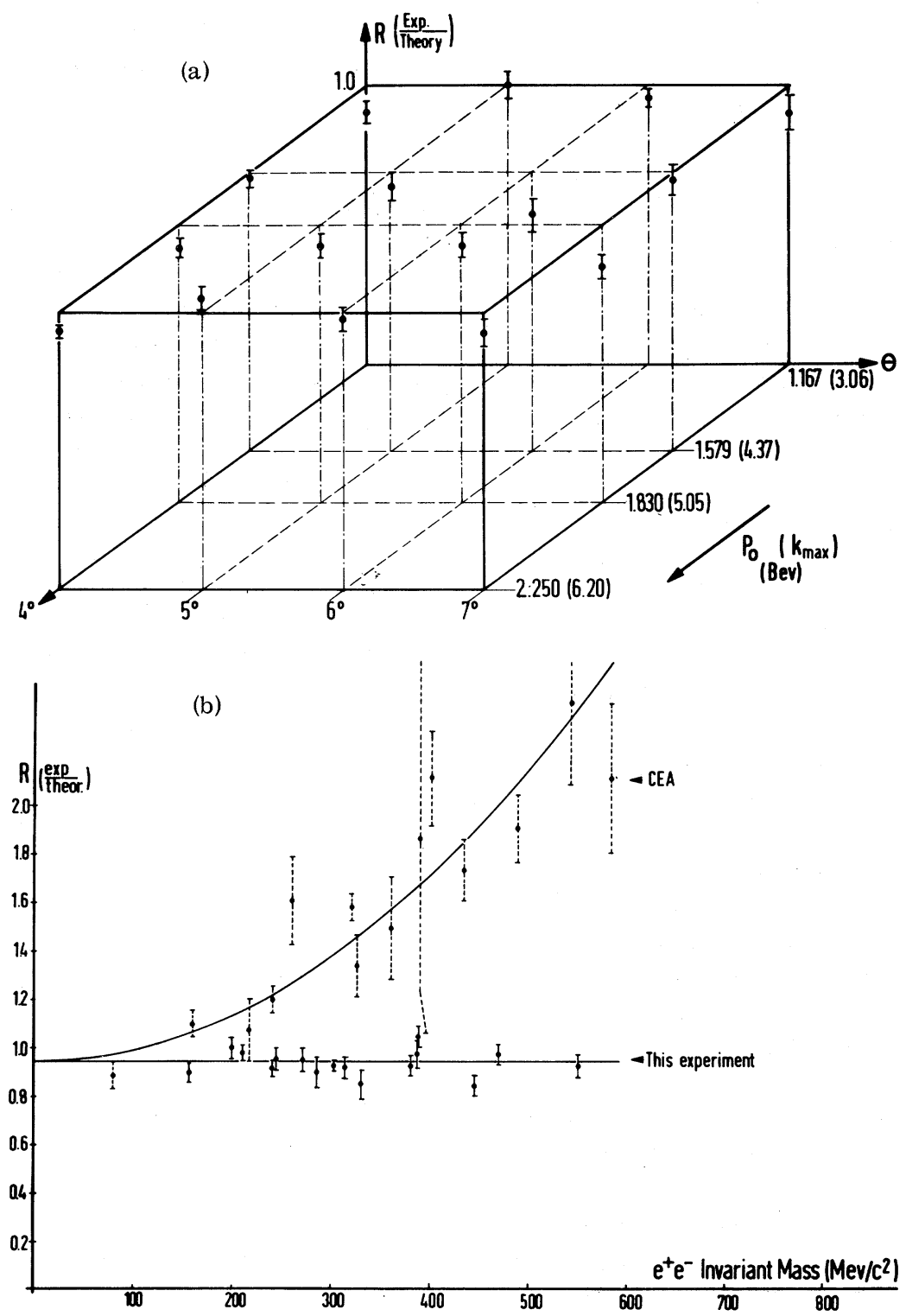

FIG. 3. Results. (a) The ratio of experiment to theory is shown for all data points. The points are arranged in a matrix, the detailed study of which (see text) shows that systematic errors in the experiment are insignificant. (b) The ratio of experiment to theory is shown as a function of the $e^{+} e^{-}$invariant mass. The straight line is the best fit to our data. Earlier work at CEA (Ref. 1) is also shown. To facilitate comparison, the normalization of the CEA data is changed in the figure so that the best-fit curve agrees with our best fit at zero pair mass. 
umn the production angle and the ratio $k / k_{\max }$ are constant ( $k$ is the central pair energy; $k_{\max }$ is the peak bremsstrahlung beam energy). Therefore, the relative yields within each column are subject to the same radiative corrections, bremsstrahlung corrections, and spectrometer acceptance. Except for multiple scattering and possible small variation with energy of the quantameter constant, the yield should follow (after form-factor corrections) a simple $1 / k^{2}$ law. In a given row, where both $k$ and $k / k_{\max }$ are constant and only the production angle is varied, the relative yields are insensitive to multiple scattering, bremsstrahlung loss, scattering from pole faces or shielding, radiative corrections, and energy dependence of the quantameter constant. The only possible systematic error could be in the calculation of the spectrometer acceptance. As seen in the figure, each point of the matrix is consistent with the prediction of QED. Thus we conclude that no significant systematic errors are present, and that the present form of QED correctly describes the production of electron-positron pairs.

Figure 3(b) shows our results (as a function of the invariant mass $M=\sqrt{2} t$ of the $e^{+} e^{-}$pair) along with other published data. The best fit of our data linear in $M$ is

$$
R=0.95 \pm 0.04-(0.4 \pm 1.1) \times 10^{-4} M,
$$

where $M$ is expressed in $\mathrm{MeV} / c^{2}$. The uncertainty in the normalization, estimated to be $5 \%$, is not included. For comparison with the Cambridge Electron Accelerator (CEA) result, we give the best fit quadratic in $M$, namely, ${ }^{12,13}$

$$
R=0.94 \pm 0.02-(5.8 \pm 15.7) \times 10^{-8} M^{2} .
$$

Both these fits are consistent with a straight line of zero slope. The CEA result, on the other hand, is

$$
R=0.67\left\{(1 \pm 0.04)+(513 \pm 38) \times 10^{-8} M^{2}\right\},
$$

which is inconsistent with our result.

Dr. S. J. Brodsky of Columbia University merits special thanks for his calculation of the radiative corrections to the yield. We are grateful for the enthusiastic support of Professor W. Jentschke and Professor P. Stähelin of DESY and Professor L. M. Lederman and Professor S. Devons of Columbia, which made this collaboration possible. We thank $\mathrm{Dr}$ 。H. O. Wüster for extending to us the DESY computing facilities, and Mr. H. Kumpfert and the synchrotron staff for their skillful operation of the ma- chine. We acknowledge the interest of Dr. H. D. Schulz in the early phases of the experiment, and the many interesting discussions with Professor H. Joos and Professor G. Weber, and Dr. F. Gutbrod, Dr. D. Schildknecht, and Dr. R. D. Kohaupt. One of us (C.C.T.) would like to thank Professor S. D. Drell and Professor N. W. Kroll for helpful discussions regarding theoretical aspects of the experiment. Lastly, we acknowledge discussions with Professor F. M. Pipkin and Professor R. Talman.

\footnotetext{
*Volkswagen Foundation Fellow at Deutsches Elektronen-Synchrotron.
}

$\dagger$ A Deutsches Elektronen-Synchrotron-Columbia Collaboration; this work was supported in part by the U.S. Atomic Energy Commission.

${ }^{1}$ R. B. Blumenthal, D. C. Ehn, W. L. Faissler, P. M. Joseph, L. J. Lanzerotti, F. M. Pipkin, and D. G. Stairs, Phys. Rev. 144, 1199 (1966). (This paper includes a complete discussion of the literature concerning pair production by photons.)

${ }^{2}$ J. D. Bjorken, S. D. Drell, and S. C. Frautschi, Phys. Rev. 112, 1409 (1958). In the text this reference is referred to as BDF.

${ }^{3}$ A. Krass, Phys. Rev. 138, B1268 (1965); S. D. Drell, in Electron and Photon Interactions at the High Energies, Invited Papers Presented at the International Symposium, Hamburg, 1965 (Springer-Verlag, Berlin, Germany, 1965), Vol. 1, p. 71.

${ }^{4}$ The $e^{+} e^{-}$yields were measured to $3 \%$ accuracy with $\mathrm{C}, \mathrm{O}, \mathrm{Al}, \mathrm{Cu}$, and $\mathrm{Pb}$ targets for a momentum transfer to the nucleus $\left\langle q^{2}\right\rangle^{1 / 2}=30 \mathrm{MeV} / c$. The relative yields show good agreement with the law $Z^{2} G_{E}{ }^{2}\left(q^{2}\right)$, where $G_{E}{ }^{2}\left(q^{2}\right)$ is the electric form factor of the target nucleus and $Z$ is the nuclear charge.

${ }^{5}$ Runs were taken for target thicknesses $T$ varying from 0.4 to $3.3 \mathrm{~g} / \mathrm{cm}^{2}$. The results show no evidence of a positive $T^{2}$ dependence of the yield.

${ }^{6}$ R. R. Wilson, Nucl. Instr. 1,101 (1957). The calibration constant for the quantameter is $(3.35 \pm 0.10)$ $\times 10^{18} \mathrm{MeV} / \mathrm{C}$, as given in Deutsches Elektronen-Synchrotron Internal Report No. DESY 65/2, 1966 (unpublished).

${ }^{7}$ The hodoscopes, used in this experiment to test properties of the spectrometer, provide resolutions $\Delta \theta / \theta$ $= \pm 0.03, \Delta t / t= \pm 0.02$, and $\Delta \psi= \pm 2.0 \mathrm{mrad}$. The hodoscopes were designed primarily for a concurrent experiment to measure the photoproduction of mesons.

${ }^{8} \mathrm{Muons}$ as well as pions were rejected by the threshold and shower counters. In the counters LC and RC the muon threshold was $3.4 \mathrm{GeV} / c$, roughly $500 \mathrm{MeV}$ above the high-momentum limit of the single-arm spectrometer acceptance. The shower counters were only $\sim 1 \%$ efficient on muons, as measured with cosmic rays. Muon contamination (including that from the decay $\pi$ $\rightarrow \mu$ ) is estimated to be $<0.1 \%$.

${ }^{9}$ For the approximate expression for $d \sigma_{\mathrm{BH}}$ see Ref. 1. ${ }^{10}$ S. D. Drell and J. D. Walecka, Ann. Phys. (N.Y.) $\underline{28}$, 18 (1964). 
${ }^{11}$ J. H. Fregeau, Phys. Rev. 104, 225 (1956).

${ }^{12}$ For a discussion of possible modifications of QED, see N. H. Kroll, CERN Report No. 66/430/5-TH, 647, 1966 (unpublished).

${ }^{13}$ For recent results concerning the production of muon pairs, see J. K. de Pagter, J. I. Friedman, G. Glass,
R. C. Chase, M. Gettner, E. von Goeler, R. Weinstein, and A. M. Boyarski, Phys. Rev. Letters 17, 767 (1966). Low-momentum-transfer measurements of $e^{+} e^{-}$pair production, also indicating a deviation from QED, were reported by R. M. Talman, Bull. Am. Phys. Soc. 11, 380 (1966).

E R R A T A

COMPOSITE MODELS OF HADRONS AND HIGH ENERGY SCATTERING. Michael Ram [Phys.

Rev. Letters 17, 1222 (1966)].

Reference 12 should have read as follows:

The essential reason for this is that the construction of particles with integral charge out of quarks requires the use of combinations of quarks with zero total triality [see M. Gell-Mann and Y. Ne'eman, The Eightfold Way (W. A. Benjamin, Inc., New York, 1964), p. 163]. Such zero-triality combinations do not contain an SU(3) triplet representation. We cannot therefore build the triplet $t$ out of quarks.

MELTING RELATIONS. Stanley E. Babb, Jr. [Phys. Rev. Letters 17, 1250 (1966)].

Footnote $\mathrm{c}$ to Table I should be associated with the $\Delta T_{65^{\circ} \mathrm{K}}$ column, and footnote d with $\Delta T_{\mathrm{m} . c}$; i.e., if the deviations from Eq. (1) are regarded as being purely temperature errors the rms deviation is $2.15^{\circ} \mathrm{K}$, or if purely volume errors the $\mathrm{rms}$ deviation is 0.006 . In a similar fashion footnote $\mathrm{b}$ of Table II should also be associated with the $\Delta T$. 